specifying and propagating CENP-A localization and centromere identity remain elusive.

Foltz et al. make the intriguing observation that the nucleolar protein, nucleophosmin-1, is associated with CENP-A chromatin. This is interesting in light of previous observations that centromeres are clustered around nucleoli during interphase in fly and human cells, that centromere proteins are present in purified nucleoli ${ }^{14}$ and that a nucleolar transcription factor interacts with CENP-C ${ }^{15}$. Previous studies demonstrated that enhancer-blocking insulators are also associated with nucleophos$\min ^{16}$. It is possible that nucleolus 'anchoring' is a conserved mechanism for sequestering centromeres and other specialized chromatin sites (Fig. 1). This interaction may be important for centromeric chromatin assembly, higher order structure, or promoting or reducing accessibility to other factors. Additional work is needed to clarify the relationship between nucleoli and centromeres. Determining how important these observations are requires direct assessment of the effects of depleting nucleolar proteins on the composition and function of centromeric chromatin, and subsequent kinetochore formation during mitosis.

These studies have identified a large number of new proteins associated with vertebrate centromeric chromatin, and demonstrated their importance to CENP-A incorporation, kinetochore formation and chromosome segregation. Future studies based on these results, and other intriguing observations, are likely to generate a more complete understanding of the spatial organization and functions of these complexes, as well as the molecular mechanisms involved in centromere identity, propagation and kinetochore assembly. However, these studies do bring us perilously close to the end of the alphabet, should future studies identify additional CENPs.
1. Cleveland, D.W., Mao, Y. \& Sullivan, K. F. Cell 112, 407-421 (2003)

2. Sullivan, K.F. Curr. Opin. Genet. Dev. 11, 182-188 (2001).

3. Blower, M. D., Sullivan, B. A. \& Karpen, G. H. Dev. Cell 2, 319-330 (2002).

4. Sullivan, B. A. \& Karpen, G. H. Nature Struct. Mol. Biol. 11, 1076-1083 (2004).

5. Mellone, B. G. \& Allshire, R.C. Curr. Opin. Genet. Dev. 13, 191-198 (2003).

6. Foltz, D.R. et al. Nature Cell Biol. 8, 458-469 (2006).

7. Okada, M. et al. Nature Cell Biol. 8, 446-457 (2006).

8. Obuse, C. et al. Genes Cells 9, 105-120 (2004).

9. Chen, E.S., Saitoh, S., Yanagida, M. \& Takahashi, K. Mol. Cell 11, 175-187 (2003)

10. Ray-Gallet, D. et al. Mol. Cell 9, 1091-1100 (2002).

11. Shelby, R. D., Monier, K. \& Sullivan, K. F. J. Cell Biol. 151, 1113-1118 (2000).

12. Takahashi, K., Chen, E. S. \& Yanagida, M. Science 288, 2215-2219 (2000)

13. Nishihashi, A. et al. Dev. Cell 2, 463-476 (2002).

14. Ochs, R. L. \& Press, R.I. Exp. Cell Res. 200, 339-350 (1992).

15. Pluta, A. F. \& Earnshaw, W. C. J. Biol. Chem. 271, 18767-18774 (1996).

16. Yusufzai, T. M., Tagami, H., Nakatani, Y. \& Felsenfeld, G. Mol. Cell 13, 291-298 (2004).

\title{
Protein expression: one by one
}

Most of what we assume to be true about gene expression is based on genetic and biochemical studies on total pools of molecules and cells, and even single-cell measurements have so far lacked the sensitivity to allow observation of protein expression at the single-molecule level. Now Sunney Xie and colleagues describe two powerful techniques that can track single protein expression, even of low-copy number proteins.

The technique reported in Nature $(440,358-362 ; 2006)$ is based on the 'veteran' gene reporter $\beta$-galactosidase ( $\beta$-gal) that is expressed from the lac $Z$ gene. Although $\beta$-gal is a highly-sensitive probe, the fluorescent molecules it produces, following substrate hydrolysis, are not retained in the cell. The authors used closed microfluidic chambers to trap the fluorescent molecules excreted by the cells in the small volume of the chambers. In doing so, they were able to obtain realtime quantitative information on gene expression in live Escherichia coli cells with single molecule sensitivity. Furthermore, they showed that this technique was also applicable to budding yeast and mouse embryonic stem cells expressing $\beta$-gal from the GAL1 or ROSA promoters, respectively.

The second technique, reported in Science (311, 1600-1603; 2006), replaces the native $l a c Z$ gene with a fusion protein of a fluorescent tag (YFP-Venus) and Tsr (a membrane protein), so it can be used as a reporter for monitoring protein expression from the lac promoter. By tracking the disappearance of the fluorescence signal after photobleaching, the authors could show that each fluorescent peak corresponded to a single molecule.

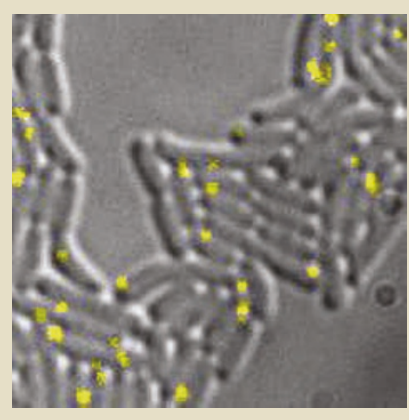

An overlay of the DIC and fluorescence images of $E$. coli cells expressing the fluorescent protein Venus, tethered to the membrane protein Tsr. Single Tsr-Venus fusion molecules (yellow spots) can be detected when they anchor to the inner membrane of the cell.

In both studies, the authors concluded that protein molecules are produced in bursts randomly occurring over time, that the number of molecules per burst follows an exponential distribution, and that each burst results from a stochastically transcribed single mRNA. Furthermore, the burst size and frequency could be determined either by real-time quantitative monitoring of protein production or by measuring the steady-state distribution of the number of protein copies within a population of cells.

Xie and colleagues have developed two highly related methods that allow single-molecule sensitivity at a single-cell level. These techniques offer new possibilities for understanding gene expression and will allow genome-wide characterization of low-copy number proteins.

MYRTO RAFTOPOULOU 